\title{
Kerr Nonlinearity Mitigation: Mid-Link Spectral Inversion Versus Digital Backpropagation in 5×28-GBd PDM 16-QAM Signal Transmission
}

Sackey, Isaac; Da Ros, Francesco; Karl Fischer, Johannes; Richter, Thomas; Jazayerifar, Mahmoud; Peucheret, Christophe; Petermann, Klaus; Schubert, Colja

Published in:

Journal of Lightwave Technology

Link to article, DOI:

10.1109/JLT.2015.2393152

Publication date:

2015

Document Version

Peer reviewed version

Link back to DTU Orbit

Citation (APA):

Sackey, I., Da Ros, F., Karl Fischer, J., Richter, T., Jazayerifar, M., Peucheret, C., Petermann, K., \& Schubert, C. (2015). Kerr Nonlinearity Mitigation: Mid-Link Spectral Inversion Versus Digital Backpropagation in 5×28-GBd PDM 16-QAM Signal Transmission. Journal of Lightwave Technology, 33(9), 1821-1827. https://doi.org/10.1109/JLT.2015.2393152

\section{General rights}

Copyright and moral rights for the publications made accessible in the public portal are retained by the authors and/or other copyright owners and it is a condition of accessing publications that users recognise and abide by the legal requirements associated with these rights.

- Users may download and print one copy of any publication from the public portal for the purpose of private study or research.

- You may not further distribute the material or use it for any profit-making activity or commercial gain

- You may freely distribute the URL identifying the publication in the public portal 


\title{
Kerr Nonlinearity Mitigation: Mid-Link Spectral Inversion versus Digital Backpropagation in 5×28-GBd PDM 16-QAM Signal Transmission
}

\author{
Isaac Sackey, Francesco Da Ros, Student Member, IEEE, Johannes Karl Fischer, Member, IEEE, \\ Thomas Richter, Student Member, IEEE, Mahmoud Jazayerifar, Christophe Peucheret, \\ Klaus Petermann, Fellow, IEEE, and Colja Schubert
}

\begin{abstract}
We experimentally investigate Kerr nonlinearity mitigation of a 28-GBd polarization-multiplexed 16-QAM signal in a 5-channel 50-GHz spaced wavelength-division multiplexing (WDM) system. Optical phase conjugation (OPC) employing the mid-link spectral inversion technique is implemented by using a dual-pump polarization-independent fiber-optic parametric amplifier (FOPA) and compared to digital backpropagation (DBP) compensation over up to $800-\mathrm{km}$ in a dispersion-managed link. In the single-channel case, the use of the DBP algorithm outperformed the OPC with a Q-factor improvement of $0.9 \mathrm{~dB}$ after 800-km transmission. However, signal transmission was not possible with DBP in the WDM scenario over the same link length while it was enabled by the OPC with a maximum Q-factor of 8.6 dB.
\end{abstract}

Index Terms - Coherent detection, fiber nonlinearity, optical phase conjugation (OPC), quadrature amplitude modulation (QAM), wavelength-division multiplexing (WDM), digital backpropagation (DBP), optical fiber communication.

\section{INTRODUCTION}

$\mathrm{T}$ RANSMISSION of advanced modulation formats and extension of transmission reach are two of the methods used to sustain the growth in global data demand. Currently, channels are tightly packed, as in dense wavelength-division

Manuscript received November 05, 2014; revised January 09, 2015; accepted January 12, 2015.

This work was supported in part by the German Research Foundation (DFG) under grants GR 3774/1-1 and PE 319/26-1; the German Federal Ministry of Education and Research under support code 16BP12310 EUREKA project SASER as well as the Danish Agency for Technology and Production Sciences under project 09-066562. The authors would like to thank OFS Denmark for providing the dispersion-managed fibers.

I. Sackey, M. Jazayerifar, and K. Petermann are with the Technische Universität Berlin, Fachgebiet Hochfrequenztechnik, Einsteinufer 25, 10587 Berlin, Germany, (e-mail: isaac.sackey@hhi-extern.fraunhofer.de)

F. Da Ros is with the Technical University of Denmark, Department of Photonics Engineering, DK-2800 Kgs. Lyngby, Denmark.

J. K. Fischer, T. Richter, and C. Schubert are with the Fraunhofer Institute for Telecommunications Heinrich Hertz Institute, Einsteinufer 37, 10587 Berlin, Germany

C. Peucheret is with the University of Rennes 1, FOTON Laboratory, CNRS UMR 6082, ENSSAT, F-22305 Lannion, France.

Color versions of one or more of the figures in this paper are available online at http://ieeexplore.ieee.org.

Copyright (c) 2015 IEEE. Personal use of this material is permitted. However, permission to use this material for any other purposes must be obtained from the IEEE by sending a request to pubs-permissions@ieee.org. multiplexing (DWDM) systems, and signal launch powers are increased in order to achieve a guaranteed optical signal-to-noise ratio (OSNR) at the receiver [1], [2]. However, power-dependent nonlinear distortion from the Kerr nonlinearity in the transmission fiber including intra- and inter-channel crosstalk and self-phase modulation degrades the transmitted signal and therefore decreases the transmission reach. It has been reported that higher-order modulation formats are more prone to Kerr nonlinearity distortions and are severely degraded in tightly packed channels [3]. Therefore advanced modulation formats (e.g. 16-quadrature amplitude modulation, 16-QAM) withstand shorter distance transmission with acceptable degradation compared to less spectrally efficient formats. Moreover, modulation formats with higher symbol rates are easily affected by dispersion-induced distortion, which can interact with nonlinear effects even over short fiber lengths.

In view of these, recent years have witnessed consistent and progressive research studies with the aim to mitigate both chromatic dispersion and Kerr nonlinear distortions in optical communication systems. Some of the reported distortion compensation schemes include digital back propagation (DBP) [4-6], optical phase conjugation (OPC) [7-11], all-optical coherent superposition using phase-sensitive amplification [12] and the so called "phase-conjugated twin waves" scheme [13-15].

The DBP algorithm relies on inverting the distortions caused by chromatic dispersion and Kerr nonlinearities digitally by the propagation of the received signal through a virtual link characterized by opposite dispersion and nonlinear coefficient with respect to the fiber link. DBP has been shown to be effective for Kerr nonlinearity mitigation especially for single-channel systems. Due to the computational complexity [16], current research is focused on reduced complexity approaches [5] as well as on novel methods for digital mitigation of inter-channel nonlinear impairments [17], [18].

Alternatively, an OPC-based technique provides compensation through mid-link spectral inversion (MLSI). In this scheme, a conjugate of the propagating signal is created at the middle of the transmission link. Propagating the conjugated signal for the remaining half of the link cancels out chromatic 
dispersion and fiber nonlinear distortions that have been accumulated in the first half of the link.

In [19], we experimentally demonstrated the implementation of the MLSI scheme for chromatic and nonlinear distortions cancellation for 50-GHz spaced channels with $5 \times 28$-GBd PDM 16-QAM signals in a $400-\mathrm{km}$ dispersion-uncompensated standard single-mode fiber (SSMF) link with backward-pumped distributed Raman pumps. A numerical model using VPItransmissionMaker v8.7 was employed to predict possible transmission reach of up to $2800 \mathrm{~km}$ using parameters which have been derived from the experiment.

As both MLSI and DBP are promising techniques, a direct comparison between the two schemes for higher order modulation format is of high interest. Such a comparison has so far only been performed numerically for dispersion-unmanaged SSMF links and non-zero dispersion-shifted fiber (NZ-DSF) links, as well as for a dispersion-managed NZ-DSF link [20].

In this work, we extend our experimental investigations of Kerr nonlinearity mitigation using MLSI and compare the results with DBP for up to $800-\mathrm{km}$ transmission in a dispersion-managed link employing super-large area (SLA) fiber and inverse dispersion-shifted fiber (IDF) with erbium-doped fiber amplifiers (EDFAs). A dual-pump polarization-independent fiber-based optical parametric amplifier (FOPA) is used as OPC device to provide mid-link spectral inversion of $5 \times 28$-GBd PDM 16-QAM signals with $50-\mathrm{GHz}$ spacing. Note that the generated conjugate signal copies in the FOPA are known as idlers. Alternatively, a split-step Fourier method (SSFM) DBP algorithm is employed for the mitigation of the fiber nonlinearities and the Q-factor (obtained from the BER) performance of the two compensation schemes is compared. In this comparison, the standard DBP based on SSFM serves as a reference benchmark for the compensation of intra-channel nonlinear impairments. Both compensation methods outperform direct transmission. However, while the DBP approach provides a better improvement for a single-channel scenario, OPC outperforms the SSFM DBP in WDM systems.

\section{EXPERIMENTAL SETUP}

The experimental set-up is depicted in Fig. 1. At the transmitter, five C-band external cavity lasers (ECLs) at wavelengths $\quad 1549.32 \mathrm{~nm}, \quad 1549.72 \mathrm{~nm}, \quad 1550.12 \mathrm{~nm}$, $1550.52 \mathrm{~nm}$ and $1550.92 \mathrm{~nm}$ (50-GHz channel spacing) were used as continuous-wave (cw) WDM signal sources. All the WDM channels were combined using an optical coupler, and after amplification with an EDFA, the signals were sent to an IQ modulator (IQ Mod). A two-channel 56-GS/s digital-to-analog converter (DAC) was used to drive the IQ Mod. This provided the in-phase and quadrature components of a single-polarization 28-GBd 16-QAM signal. After using a polarization-multiplexing emulator (PolMux) to provide PDM signals to all five WDM channels, the channels were decorrelated. A wavelength-selective switch (WSS) was used to separate all the channels and different lengths of single-mode fiber patch cords were placed in the individual WDM optical paths. This provided a minimum inter-channel delay of 100 symbols [21]. An EDFA was used to amplify the channels after combining all five WDM signals with an optical coupler. The out-of-band amplified spontaneous emission (ASE) noise was filtered by using a 3 -nm optical band-pass filter (OBPF) and the state-of-polarization of the data was randomized using a polarization scrambler.

The transmission link consists of $80-\mathrm{km}$ dispersion-compensated spans made from SLA and IDF fibers. The specifications of a representative span can be seen in Table 1 in section IV of the paper. An EDFA was used in front

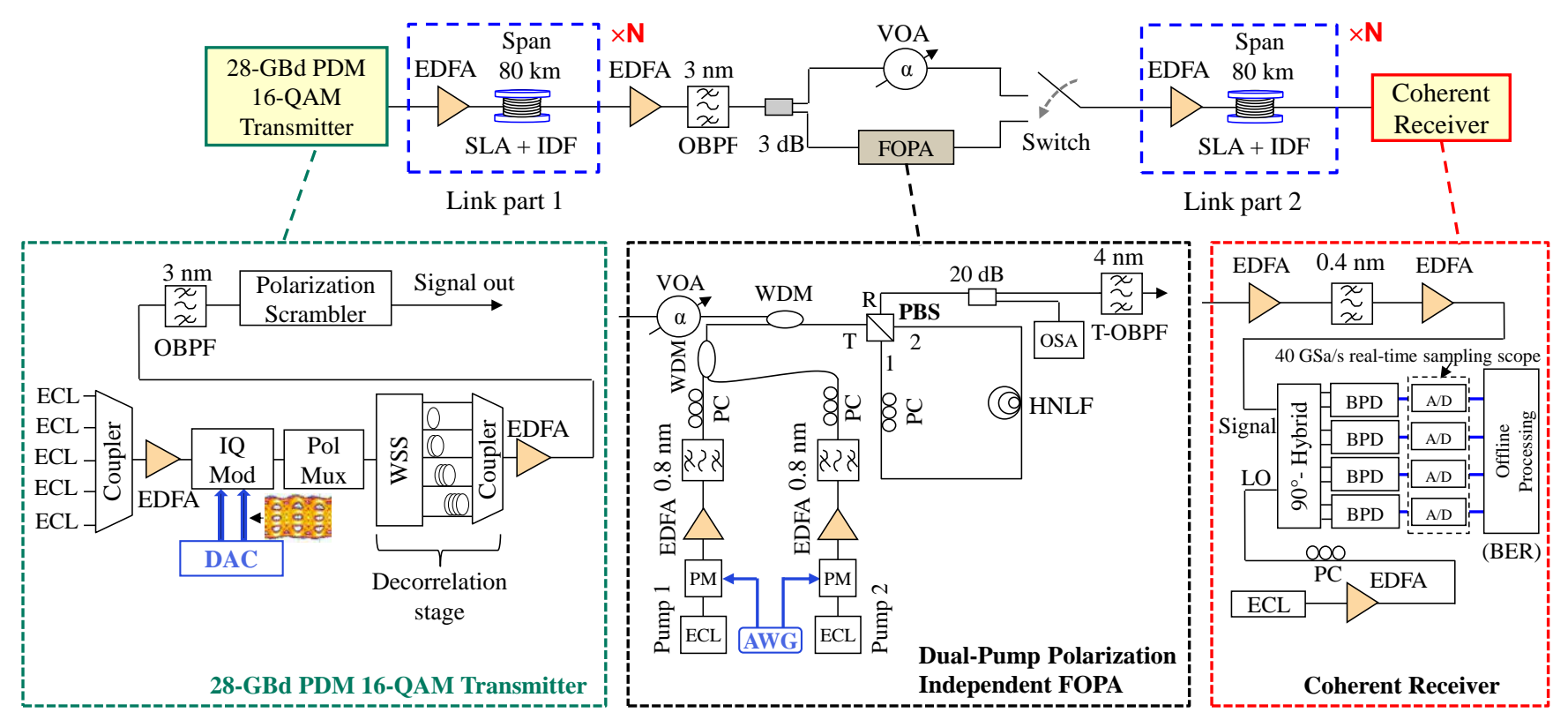

Fig. 1. Experimental setup showing the $5 \times 28$-GBd 16-QAM transmitter, the dispersion-managed fiber links (SLA+IDF), the dual-pump polarization-independent FOPA, which is used as OPC device, and the coherent receiver. 
of each fiber span to set the signal launch power. After the first half of the link ( $\mathrm{N}$ spans in link part 1), an EDFA was set to a constant output power of $17 \mathrm{dBm}$ so as to keep the input signal power into the FOPA constant. A 3-nm OBPF was used to suppress the accumulated ASE and the signal was split into two portions with a 3-dB optical coupler. One portion of the signal went through the OPC device whereas the other portion bypassed the OPC device, which is referred to, here, as 'direct' transmission. The design and performance evaluation of the OPC device is discussed in section III. A variable optical attenuator (VOA) in the path without OPC ensured that the same input power was injected to the first EDFA of link part 2 in both scenarios, thereby resulting in the same noise accumulation behavior in the second half of the link. As the OPC provided low-loss operation, the additional attenuation introduced by the VOA in the path without OPC was about $5 \mathrm{~dB}$ and it was mainly due to the relatively high loss of the OBPF ( $5 \mathrm{~dB}$ ) used to select the idler at the OPC output. Note that a conventional link without OPC would not consist of the interface between the link part 1 and link part 2 in Fig. 1. However, this interface has a negligible effect on the noise performance of the link since as mentioned before, the first EDFA in the interface is set to a large constant output power and the signal level is high during the optical processing in the interface and reduces only to about $2 \mathrm{dBm}$ measured at the input of the first EDFA in link part 2. Therefore, the interface causes a negligible OSNR penalty. An optical switch was used to either select the output of the OPC device (conjugated signal) or the signal which bypassed the OPC device (direct transmission) before launching the selected data into the remaining half of the link ( $\mathrm{N}$ spans in link part 2).

Detection of the signal or the conjugated copy after transmission over the entire link was performed with a standard polarization-diverse coherent receiver using a local oscillator (LO) with $100-\mathrm{kHz}$ linewidth. The LO was combined with the selected data (either the signal or the conjugated signal) after

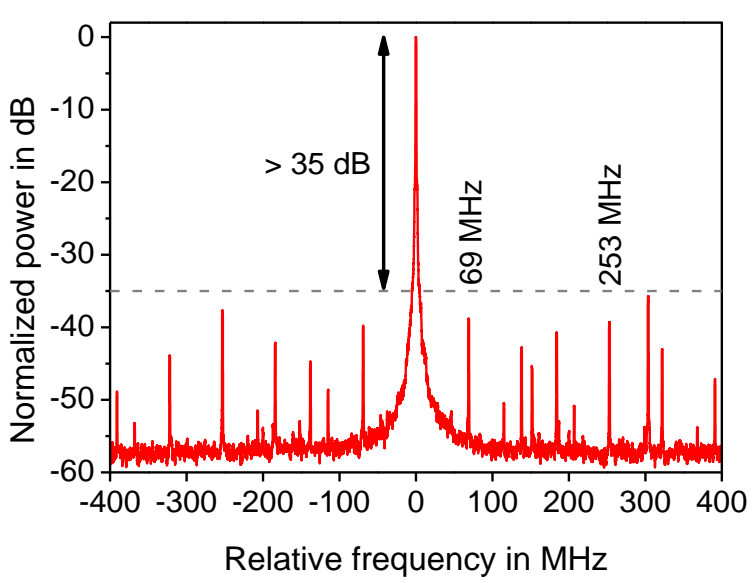

(a) the transmission link in a $90^{\circ}$ optical hybrid. Four balanced photo detectors (BPD) were connected to the hybrid outputs, and a real-time sampling scope (RTO, 40-GS/s sampling rate, 20-GHz bandwidth) was used as analog-to-digital converter (A/D). Offline processing was performed on a desktop computer including resampling, $90^{\circ}$ optical hybrid correction, frequency-offset compensation, blind adaptive time-domain equalization using a constant-modulus algorithm and multi-modulus algorithm, carrier-phase estimation by blind phase search, de-mapping and bit-error counting. Note that, with the exception of the transmission link; the transmitter, the OPC device and the coherent receiver used in this investigation are the same as those used in [19].

\section{Characterization of The OPC Device}

The schematic representation of the dual-pump polarization-independent FOPA that was used as the OPC device is shown in Fig. 1. The OPC device consists of two ECLs at wavelengths $1534 \mathrm{~nm}(25-\mathrm{kHz}$ linewidth $)$ and $1574 \mathrm{~nm}(100-\mathrm{kHz}$ linewidth), which served as cw pump sources. Two sinusoidal radio frequency (RF) tones at frequencies $69 \mathrm{MHz}$ and $253 \mathrm{MHz}$ were generated by a two-channel arbitrary waveform generator (AWG). The pumps were independently phase-modulated, with a modulation index of 1.44 radian, using the AWG output waveforms via phase modulators (PMs). The PMs were driven in a counter-phasing fashion in order to minimize the transfer of pump phase modulation from the pump to the generated idlers [22], [23]. The pumps were amplified by two EDFAs, filtered and combined with a WDM coupler. OBPFs with 0.8-nm full-width at half maximum bandwidths were used to suppress the out-of-band ASE noise around the pumps. The signal and the pumps were then sent to the highly nonlinear fiber (HNLF) inside the polarization diversity loop via a polarization beam splitter (PBS). Polarization controllers (PCs) in the pump paths were used to equally split the pumps in both propagation

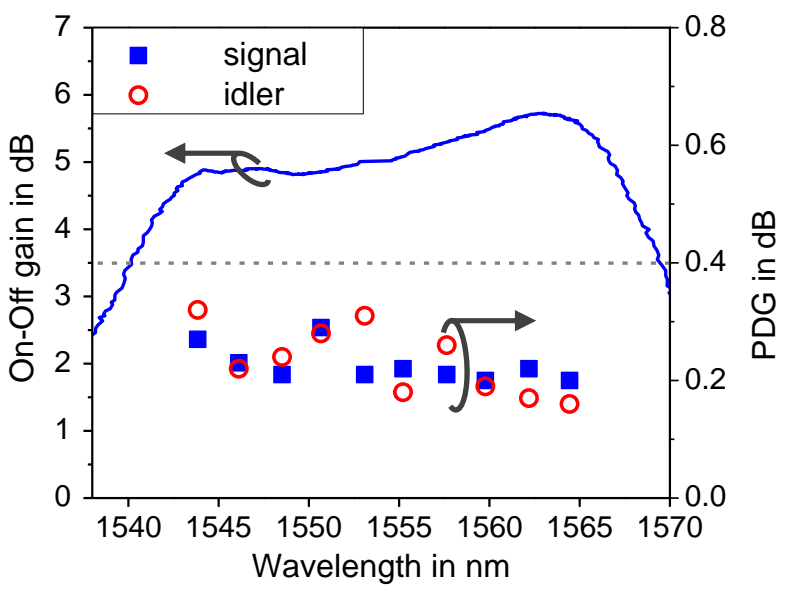

(b)

Fig. 2. (a) Acquired electrical spectrum from RTO showing suppression of pump phase modulation (b) On-off gain profile and PDG performances of both signal and idler. 


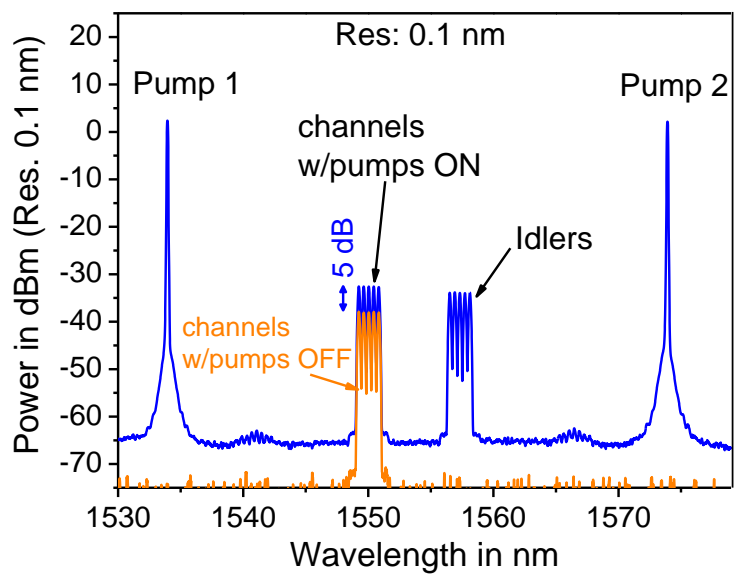

(a)

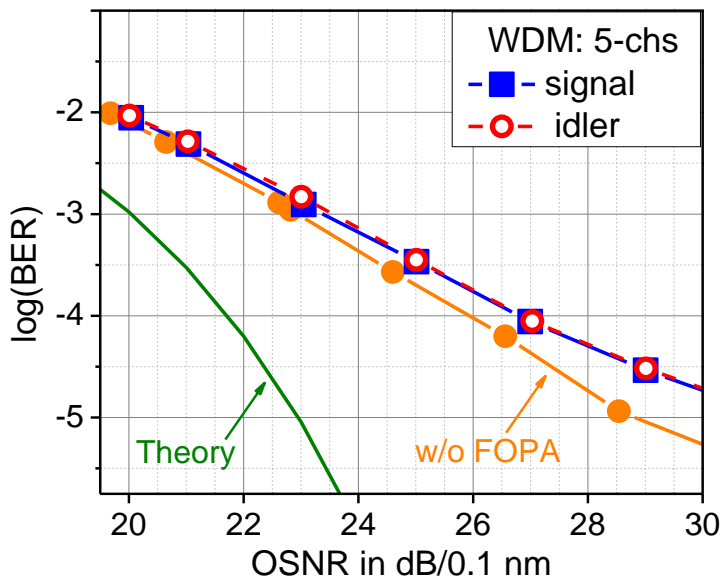

(b)

Fig. 3. (a) 5-channel WDM spectrum measured after a 20-dB coupler showing the on-off gain, (b) Plot of BER vs OSNR for the WDM scenario showing the performance of the signal and the idler.

directions of the loop. The HNLF length, nonlinear coefficient, zero-dispersion wavelength, dispersion slope, and attenuation were $300 \mathrm{~m}, 16.3 / \mathrm{W} / \mathrm{km}, 1556.6 \mathrm{~nm}, 0.019 \mathrm{ps} / \mathrm{nm}^{2} / \mathrm{km}$, and $1.4 \mathrm{~dB} / \mathrm{km}$, respectively. Operating the OPC in an unsaturated regime was essential to avoid signal degradation, thus the VOA at the input of the OPC was set to ensure an appropriate signal power into the HNLF [24], [25]. Note that, in order to keep the OPC penalty low, a total pump power of $28.2 \mathrm{dBm}$ and a total signal power of $-5 \mathrm{dBm}$ (for either single-channel or WDM case) were launched into the diversity loop via the PBS. This provided 5-dB signal on-off gain and 4-dB idler conversion efficiency at the output of the OPC [25], [26]. Note that a total input signal power of $-5 \mathrm{dBm}$ was used only for the back-to-back characterization of the FOPA. For the transmission experiment, however, the input signal power was optimized to $2 \mathrm{dBm}$. A 4-nm tunable-OBPF was used to filter out the channel(s) of interest at the output of the OPC. The performance evaluation of the OPC device was first carried out in the back-to-back (b2b) configuration without the transmission link.

In order to analyze the transferred pump-phase modulation to the generated idler, a single-channel $\mathrm{cw}$ signal at $1550.12 \mathrm{~nm}$ was injected together with the pumps into the diversity loop operated in a polarization-independent fashion. The strength of the phase modulation being transferred from the pumps to the idler was monitored via the electrical spectrum of the received idler measured using the RTO. While monitoring the idler spectrum, the phase delay and amplitude parameters of the pump phase modulation tones were adjusted so as to minimize the transferred pump-phase modulation to the idler. Fig. 2(a) shows the electrical spectrum of the received idler and highlight a suppression ratio between the carrier and the modulation tones higher than $35 \mathrm{~dB}$, indicating that only negligible phase modulation is transferred to the idler [22], [27].

A flat gain-bandwidth profile of the OPC device is desirable for WDM systems. To determine the gain profile, the signal-pump WDM coupler in the setup was temporarily replaced with a $10-\mathrm{dB}$ coupler. The on-off gain was measured with a polarization scrambled $\mathrm{cw}$-signal with its wavelength swept from $1535 \mathrm{~nm}$ to $1572 \mathrm{~nm}$. Fig. 2(b) shows the measured gain profile. The wavelengths of the WDM channels were chosen in the flat gain region. In order to minimize the Raman effect, which causes longer wavelengths to experience more gain than shorter wavelengths, the C-band pump power was set $\sim 1.8 \mathrm{~dB}$ higher than the L-band pump power. Fig. 2(b) also shows the measured polarization-dependent gain (PDG) of the OPC. Using a zero-span function of the optical spectrum analyzer (OSA) while scrambling the state-of-polarization of a single-channel 28-GBd 16-QAM signal with the PDM emulator bypassed, the PDG was measured for a wavelength sweep from $1542.5 \mathrm{~nm}$ to $1565 \mathrm{~nm}$ (i.e. within the flat region of the gain profile). The maximum PDG for both signal and idler was found to be below $0.4 \mathrm{~dB}$ (indicated by the dashed line).

The on-off gain of the signal as well as the idler conversion efficiency is shown in Fig. 3(a). The spectrum was obtained from an OSA after a 20-dB coupler.

The BER performance as a function of receiver OSNR for both signals and idlers in a WDM scenario is shown in Fig. 3(b). Note that the center channel (ch-3) was used for evaluations in the WDM investigations. It can be seen that, the performances of the signal and idler are very similar. This indicates the effectiveness of the suppression of the pump phase modulation transfer to the idler. It was also noted that the differences in the BER between the WDM channels were insignificant, showing similar performances for all WDM channels [27] and therefore making the OPC well applicable for WDM transmission system investigations.

\section{DigitAl BACKPROPAGATION}

A non-iterative symmetric SSFM based on coupled nonlinear Schrödinger equations (NLSEs) was employed for the realization of the DBP [5], [16]. The dispersion-managed fibers 
TABLE 1

SPECIFICATIONS OF THE DISPERSION-MANAGED FIBER (EACH SPAN CONSISTS OF SLA AND IDF FIBERS)

\begin{tabular}{lll}
\hline \hline \multicolumn{1}{c}{ Parameter (properties at $1550 \mathrm{~nm})$} & SLA & IDF \\
\hline Length $(\mathrm{km})$ & 55.57 & 25.68 \\
Effective area $\left(\mu \mathrm{m}^{2}\right)$ & 107 & 31 \\
Loss coefficient $(\mathrm{dB} / \mathrm{km})$ & 0.186 & 0.234 \\
Nonlinear refractive index $\left(\times 10^{-20} \mathrm{~m}^{2} / \mathrm{W}\right)$ & 2.23 & 2.36 \\
Dispersion $(\mathrm{ps} / \mathrm{nm} / \mathrm{km})$ & 20.2 & -44 \\
Dispersion slope $\left(\mathrm{ps} / \mathrm{nm}^{2} / \mathrm{km}\right)$ & 0.06 & -0.13 \\
\hline
\end{tabular}

were modeled using the measured parameters from the fibers used in the experiment. An example of the specifications of a fiber span is shown in Table 1. Group velocity dispersion of first and second-order were taken into account in the DBP. However, ASE noise from the link EDFAs was not taken into account [16]. Polarization mode dispersion in the link was also not considered.

The SLA and IDF fibers in each span were modeled separately by performing the SSFM on each fiber type. Each fiber was modeled with a number of $m$ steps in the SSFM DBP algorithm. The value of $m$ was optimized on a 480-km transmission scenario under WDM operation. Increasing the number of steps per fiber beyond $m=10$ did not yield further improvements. Therefore the number of steps per fiber was kept fixed at $m=10$ and corresponds to 10 steps per SLA and 10 steps per IDF. A higher number of steps can improve the DBP performance up to a certain level at the expense of higher complexity and computational effort [5], [20].

The SSFM DBP algorithm was performed on the center channel of the received 5×28-GBd PDM 16-QAM signal and the results were compared to those obtained from the OPC operation. Note that two million samples were processed in both OPC measurements and DBP computation.

\section{RESULTS AND DISCUSSIONS}

\section{A. Single-channel scenario}

We initially investigated the nonlinearity compensation performance by using the OPC with a single-channel at $1550.12 \mathrm{~nm}$ (center channel in the WDM experiment) over a transmission length of $480 \mathrm{~km}$ (i.e. $\mathrm{N}=3$ spans before and 3 spans after the OPC) and the results were compared with that of the DBP.

The received data of the direct signal transmission was backpropagated in the modeled DBP. It is seen from Fig. 4 that the maximum Q-factor for the case with DBP increases to $11.8 \mathrm{~dB}$ (at a launch power of $1 \mathrm{dBm} / \mathrm{pol}$ ). This indicates an improvement in performance of $0.8-\mathrm{dB}$ compared to the maximum Q-factor for the OPC compensation technique.

The transmission length was then increased to $800 \mathrm{~km}$ (i.e. $\mathrm{N}=5$ spans before and 5 spans after the OPC) and the calculated Q-factors were plotted as a function of the signal launch power per polarization as shown in Fig. 5. It can be seen that direct transmission without any nonlinearity mitigation

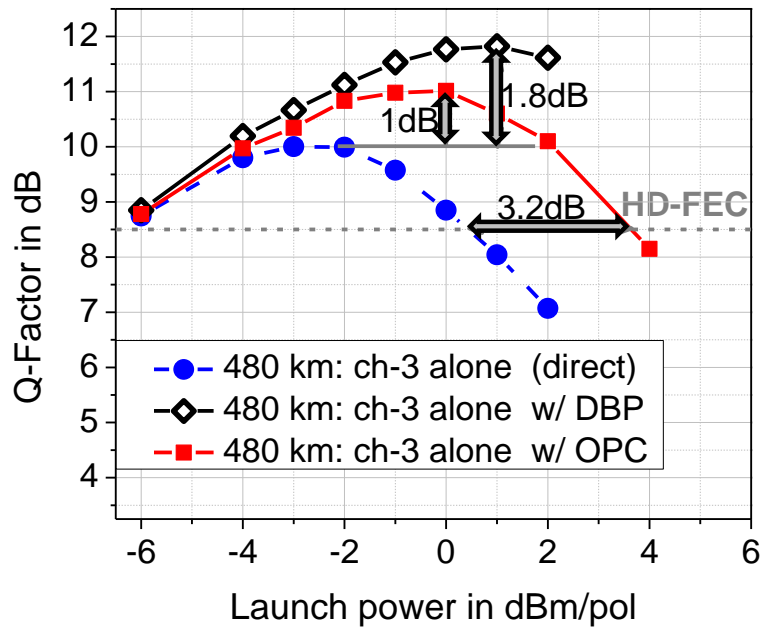

Fig. 4. Single-channel: plot of Q-factor vs. launch power per polarization over the 480-km transmission link showing the performances for the cases of direct transmission without nonlinearity mitigation, with OPC operation and with SSFM DBP.

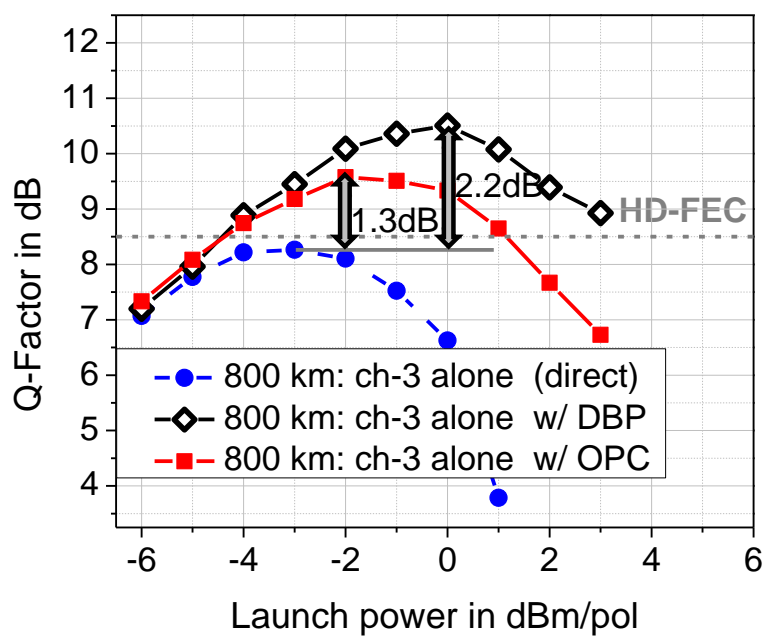

Fig. 5. Single-channel: plot of Q-factor vs. launch power per polarization over the $800-\mathrm{km}$ transmission link showing the performances for the cases of direct transmission without nonlinearity mitigation, with OPC operation and with SSFM DBP.

scheme was not possible with BERs below the hard-decision forward error correction (HD-FEC) threshold of $3.8 \times 10^{-3}$, corresponding to a Q-factor of $8.5 \mathrm{~dB}$. However, with the implementation of the OPC the maximum Q-factor increased from $8.3 \mathrm{~dB}$ to $9.6 \mathrm{~dB}$ resulting in a $\mathrm{Q}$-factor improvement of $1.3 \mathrm{~dB}$. Moreover, application of the DBP increased the maximum Q-factor to $10.5 \mathrm{~dB}$. This yields an improvement of the maximum Q-factor improvement of $2.2 \mathrm{~dB}$ when compared to the direct signal transmission and is $0.9 \mathrm{~dB}$ better than the OPC scheme. The nonlinear threshold is also seen to be increased considerably thanks to the use of the DBP algorithm.

It is observed that the OPC compensation scheme shows poorer performance than the DBP. This can be attributed to the asymmetric power evolution over the entire EDFA-based 
transmission link [7], [16]. In addition, locating the OPC exactly in the middle of the link can be very difficult and therefore any asymmetry regarding the position of the OPC will affect the performance [7], [11]. The DBP algorithm, however, does not have such constraints.

In addition, the generation of an idler at a different wavelength in the OPC scheme can result in a dispersion mismatch between signal and idler which can lead to inefficient distortion compensation. However, in our experiment, the signal/idler residual dispersion mismatch was very low $(\sim 0.8 \mathrm{ps} / \mathrm{nm}$ over $400 \mathrm{~km})$ since the wavelength separation between signal and idler was only $7.2 \mathrm{~nm}$ (signal at $1550.12 \mathrm{~nm}$, idler at $1557.32 \mathrm{~nm}$ ) and also due to the use of the dispersion-managed link.

\section{B. WDM scenario}

Nonlinear distortion mitigation was also considered for a 5-channel WDM scenario. All five WDM channels were propagated over a transmission length of $480 \mathrm{~km}$. The signal launch power per channel per polarization was adjusted from $-7 \mathrm{dBm}$ to $0 \mathrm{dBm}$ and the center channel (i.e. at $1550.12 \mathrm{~nm}$ ) was evaluated in the WDM investigations. The Q-factors were calculated for each launch power from the BERs, as in the single-channel scenario, and were plotted as function of the signal launch powers as shown in Fig. 6. Direct transmission over the link produced a maximum Q-factor of $9.4 \mathrm{~dB}$ at a launch power of $-5 \mathrm{dBm} / \mathrm{ch} / \mathrm{pol}$. With the application of the $\mathrm{DBP}$, the maximum Q-factor improved to $9.9 \mathrm{~dB}$. However, implementation of the OPC increased the maximum Q-factor to $10.1 \mathrm{~dB}$. This indicates an improvement of the maximum
Q-factor of $0.7 \mathrm{~dB}$ compared to the direct transmission without any compensation scheme. Fig. 6 also shows that OPC increases the nonlinear threshold by $0.8 \mathrm{~dB}$ compared to DBP and by $2 \mathrm{~dB}$ compared to the direct transmission.

The transmission length was further increased to $800 \mathrm{~km}$ and the Q-factor performances for the three cases are shown in Fig. 7. It is clear from Fig. 7 that without the implementation of any nonlinear compensation scheme, the maximum Q-factor obtained from direct transmission was $7.7 \mathrm{~dB}$, well below the HD-FEC threshold. Even though the use of the DBP algorithm improves the maximum Q-factor to $8.2 \mathrm{~dB}$, this is still below the HD-FEC threshold. On the other hand, OPC operation increases the maximum Q-factor to $8.6 \mathrm{~dB}$, which is above the HD-FEC threshold. This implies that the signal transmission over $800 \mathrm{~km}$ was only possible with OPC operation with a $\mathrm{Q}$-factor improvement of $0.4 \mathrm{~dB}$ compared to DBP.

These results show that, in the WDM scenario, the OPC outperforms the DBP algorithm. The lower performance of the DBP in the WDM scenario is due to the detection bandwidth of the employed coherent reception which was limited to a single WDM channel. It thus compensates for intra-channel distortions but not for inter-channel distortions [28]. The OPC on the other hand, compensates for both intra- and inter-channel impairments. The same behavior has been observed for a different dispersion-managed link configuration in numerical simulations [20].

The performance of the OPC might be further improved in the WDM case over the DBP algorithm if the power profile was made more symmetrical with respect to the middle of the transmission link, a condition which is very critical to attain by using only EDFAs [7].

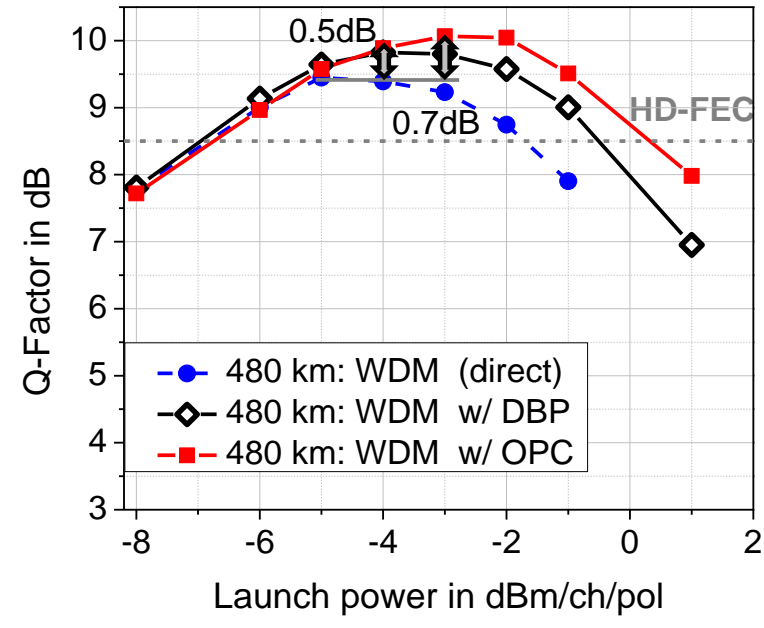

(a) (i)

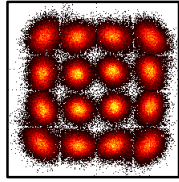

(ii)

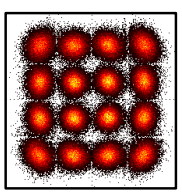

(iii)

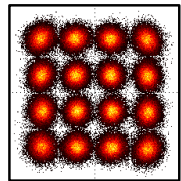

(b)

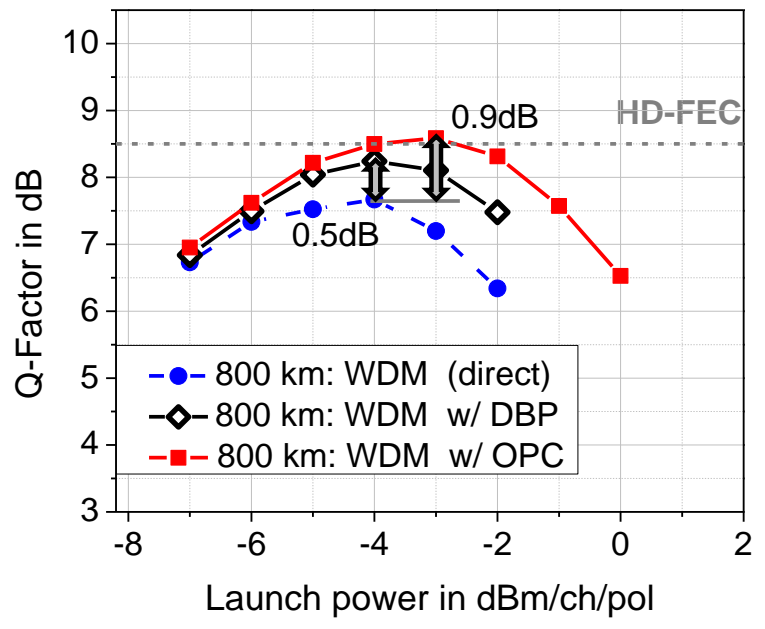

(c)

Fig. 6. (a) 5-channel WDM: plot of Q-factor vs. launch power per channel per polarization over the 480-km transmission link showing the performances for the cases of direct transmission without nonlinearity mitigation, with OPC operation and with SSFM DBP, (b) X-polarization constellation diagrams at a launch power of $-3 \mathrm{dBm} / \mathrm{ch} /$ pol over $800 \mathrm{~km}$ for the cases (i) direct transmission without any compensation scheme, (ii) with DBP, (iii) with OPC operation, (c) 5-channel WDM: plot of Q-factor vs. launch power per channel per polarization over the $800-\mathrm{km}$ transmission link showing the performances for the cases of direct transmission without nonlinearity mitigation, with OPC operation and with SSFM DBP. 


\section{CONCLUSION}

We have experimentally investigated fiber nonlinearity mitigation of a five-channel 28-GBd PDM 16-QAM signal in a 50-GHz spaced WDM system. Two nonlinear compensation methods, optical phase conjugation (OPC) based mid-link spectral inversion and digital backpropagation (DBP) were implemented and the results were compared for up to $800-\mathrm{km}$ transmission over dispersion-managed transmission links. The OPC compensation scheme shows an inferior performance compared to DBP in the single-channel case. However, in the WDM scenario over the same link length, the OPC-based compensation scheme outperformed DBP. This is due to the fact that the DBP algorithm can practically compensate for only intra-channel cross-talk, due to receiver bandwidth limitations, whereas the OPC compensates for both inter- and intra-channel impairments. Thus in our experiment, the optical domain signal processing proves to be a better approach than its digital domain counterpart for nonlinearity compensation of WDM signals.

\section{REFERENCES}

[1] P. J. Winzer, A. H. Gnauck, S. Chandrasekhar, S. Draving, J. Evangelista, and B. Zhu, "Generation and 1200-km transmission of 448-Gb/s ETDM 56-Gbaud PDM 16-QAM using a single I/Q Modulator," in Proc. Eur. Conf. Opt. Commun., (ECOC), Torino, Italy, paper PD 2.2, 2010.

[2] R. -J. Essiambre, G. Kramer, P. J. Winzer, G. J. Foschini, and B. Goebel, "Capacity limits of optical fiber networks," J. Lightw. Technol., vol. 28, no.4, pp. 662-701, Feb. 2010.

[3] C. Behrens, R. I. Killey, S. J. Savory, M. Chen, and P. Bayvel, "Nonlinear distortion in transmission of higher order modulation formats," IEEE Photon. Technol. Lett. vol. 22, no. 15, pp. 1111-1113, 2012.

[4] E. Ip, "Nonlinearity compensation using backpropagation for polarization-multiplexed transmission", J. Lightw. Technol., vol. 28, no 6 , pp. 939-950, Mar. 2010.

[5] A. Napoli, Z. Maalej, V. A. J. M. Sleiffer, M. Kuschnerov, D. Rafique, E. Timmers, B. Spinnler, T. Rahman, L. D. Coelho, and N. Hanik, "Reduced complexity digital back-propagation methods for optical communication systems, "J. Lightw. Technol., vol. 32, no. 7, pp. 1351-1362, Apr. 2014.

[6] L. Du, B. Schmidt, and A. Lowery, "Efficient digital backpropagation for PDM-CO-OFDM optical transmission systems," in Proc. Optical Fiber Commun. Conf. (OFC), paper OThE2, 2010.

[7] S. L. Jansen, D. van den Borne, P. M. Krummrich, S. Spälter, G. -D. Khoe and H. de Waardt, "Long-haul DWDM transmission systems employing optical phase conjugation," IEEE J. Sel. Topics Quantum Electron. vol. 12 , no. 4 , pp. 505-520, 2006.

[8] H. Hu, R. M. Jopson, A. H. Gnauck, M. Dinu, S. Chandrasekhar, X. Liu, C. Xie, M. Montoliu, S. Randel, and C. J. McKinstrie, "Fiber nonlinearity compensation of an 8-channel WDM PDM-QPSK signal using multiple phase conjugations," in Proc. Optical Fiber Commun. Conf. (OFC), CA, paper M3C.2, 2014.

[9] M. Morshed, A. J. Lowery, and L. B. Du, "Improving performance of optical conjugation by splitting the nonlinear element," Opt. Express, vol. 21 , pp. 4567-4577, 2013.

[10] F. Da Ros, I. Sackey, R. Elschner, T. Richter, C. Meuer, M. Nölle, M. Jazayerifar, K. Petermann, C. Peucheret, and C. Schubert, "Kerr nonlinearity compensation in a 5x28-GBd PDM 16-QAM WDM system using fiber-based optical phase conjugation," in Proc. Eur. Conf. Opt. Commun., (ECOC), Cannes, France, paper P.5.3, 2014.

[11] S. Watanabe, M. Shirasaki, "Exact compensation for both chromatic dispersion and Kerr effect in a transmission fiber using optical phase conjugation, " J. Lightw. Technol., vol. 14, no.3, pp. 243-248, Mar. 1996.

[12] S. L. I. Olsson, T. A. Eriksson, C. Lundström, M. Karlsson, and P. A. Andrekson, "Linear and nonlinear transmission of 16-QAM Over $105 \mathrm{~km}$ phase-sensitive amplified link," in Proc. Optical Fiber Commun. Conf. (OFC), CA, paper Th1H.3, 2014.

[13] X. Chen, X. Liu, S. Chandrasekhar, B. Zhu, and R. W. Tkach, "Experimental demonstration of fiber nonlinearity mitigation using digital phase conjugation," in Proc. Optical Fiber Commun. Conf. (OFC), CA, paper OTh3C.1, 2012.

[14] X. Liu, S. Chandrasekhar, P. J. Winzer, R. W. Tkach, and A. R. Chraplyvy, "Fiber-nonlinearity-tolerant superchannel transmission via nonlinear noise squeezing and generalized phase-conjugated twin waves," J. Lightw. Technol., vol. 32, no. 4, pp. 766-775, Feb. 2014.

[15] Y. Tian, Y. -K. Huang, S. Zhang, P. R. Prucnal, and T. Wang, "Demonstration of digital phase-sensitive boosting to extend signal reach for long-haul WDM systems using optical phase-conjugated copy," Opt. Express, vol. 21, pp. 5099-5106, 2013.

[16] E. Ip, and J. M. Kahn, "Compensation of dispersion and nonlinear impairments using digital backpropagation, "J. Lightw. Technol., vol. 26, no. 20 , pp. 3416-3425, Oct. 2008

[17] G. Liga, T. Xu, A. Alvarado, R. I. Killey, and P. Bayvel, "On the performance of multichannel digital backpropagation in high-capacity long-haul optical transmission, " Opt. Express, vol. 22, pp. 30053-30062, 2014.

[18] M. Shtaif, R. Dar, A. Mecozzi, and M. Feder, "Nonlinear interference noise in WDM systems and approaches for its cancelation," in Proc. Eur. Conf. Opt. Commun. (ECOC), Cannes, France, paper We.1.3.1, 2014.

[19] I. Sackey, F. Da Ros, M. Jazayerifar, T. Richter, C. Meuer, M. Nölle, L. Molle, C. Peucheret, K. Petermann, and C. Schubert, "Kerr nonlinearity mitigation in 5x28-GBd PDM 16-QAM signal transmission over a dispersion-uncompensated link with backward-pumped distributed Raman amplification," Opt. Express, vol. 22, pp. 27381-27391, 2014.

[20] D. Rafique, and A. Ellis, "Nonlinearity compensation in multi-rate 28 Gbaud WDM systems employing optical and digital techniques under diverse link configurations," Opt. Express, vol. 19, pp. 16919-16926, 2011

[21] S. K. Ibrahim, J. Zhao, F. C. G. Gunning, P. Frascella, F. H. Peters, and A. D. Ellis, "Towards a practical implementation of coherent WDM: analytical, numerical, and experimental studies, "IEEE Photon. J., vol. 2, no. 5, pp. 833-847, Oct. 2010.

[22] T. Richter, R. Elschner, A. Gandhi, K. Petermann, and C. Schubert, "Parametric amplification and wavelength conversion of single- and dual-polarization DQPSK signals," IEEE J. Sel. Topics Quantum Electron. vol. 18, no. 2, pp. 988-995, 2012.

[23] S. Radic, C. J. McKinstrie, R. M. Jopson, J. C. Centanni, A. R. Chraplyvy, C. G. Jorgensen, K. Brar, and C. Headley, "Selective suppression of idler spectral broadening in two-pump parametric architectures," IEEE Photon. Technol. Lett. vol. 15, no. 5, pp. 673-675, 2003.

[24] F. Da Ros, R. Borkowski, D. Zibar, and C. Peucheret, "Impact of Gain Saturation on the Parametric Amplification of 16-QAM Signals," in Proc. Eur. Conf. Opt. Commun., (ECOC), Amsterdam, The Netherlands, paper We.2.A.3, 2012.

[25] I. Sackey, R. Elschner, M. Nölle, T. Richter, L. Molle, C. Meuer, M. Jazayerifar, S. Warm, K. Petermann, and C. Schubert, "Characterization of a fiber-optical parametric amplifier in a $5 \times 28$-GBd 16-QAM DWDM system," in Proc. Optical Fiber Commun. Conf. (OFC), CA, paper W3E.3, 2014.

[26] M. Jazayerifar, I. Sackey, R. Elschner, S Warm, C. Meuer, C. Schubert, and K. Petermann, "Impact of SBS on polarization-insensitive single-pump optical parametric amplifiers based on a diversity loop scheme," in Proc. Eur. Conf. Opt. Commun., (ECOC), Cannes, France, paper Tu.4.6.4, 2014.

[27] I. Sackey, F. Da Ros, T. Richter, R. Elschner, M. Jazayerifar, C. Meuer, C. Peucheret, K. Petermann, and C. Schubert, "Design and performance evaluation of an OPC device using a dual-pump polarization-independent FOPA," in Proc. Eur. Conf. Opt. Commun., (ECOC), Cannes, France, paper Tu.1.4.4, 2014.

[28] S. J. Savory, G. Gavioli, E. Torrengo, and P. Poggiolini, "Impact of interchannel nonlinearities on a split-step intrachannel nonlinear equalizer," IEEE Photon. Technol. Lett. vol. 20, no. 10, pp. 673-675, 2010.

Author biographies are not included by author request due to space constraints. 